\title{
Delivering neutrons to instruments with effectiveness and efficiency
}

The low power of the $1 \mathrm{MW}$ reactor installed at Garching in the late 50s limited very much the range of experiments. In 1961, Peter Höhne and Tasso Springer deliberately placed a brass tube around a neutron beam that was left unprotected over several meters for an experiment and observed a great increase in neutron intensity at the far end of the tube [1,2]. Heinz Maier-Leibnitz, who was placing an emphasis on developing techniques, decided to push forward this principle and invented the neutron guide with Tasso Springer.

Ten years later, the neutron guides and the cold source, two novel key features at that time, enabled the Institut Laue-Langevin (ILL) to outperform all other sources. Since then, these components are widely used and numerous teams worldwide pursue the developments of new concepts and improved techniques. For some years, these teams meet regularly at the International workshop on Neutron Delivery Systems (NDS) and the 5th edition was organised from the 25 th to the 27 th of June 2018 at the ILL, Grenoble (France).

Advances in neutron technologies are often communicated verbally at meetings and can be lost. This must be avoided and we are glad to present in this issue a selection of technical notes on progress related with cold sources and neutron guides which were presented at the NDS 2018 event:

- a proposition to build an efficient cold-neutron reflector employing nanoparticles of low-absorbing highlyscattering materials,

- the status of the ultra-cold neutron sources at PSI and TRIUMF,

- a new imaging nested-mirror optics concept allowing to tailor beam properties and match instrument needs,

- an overview of the neutron guides replacement of the ILL,

- a proposition for protecting supermirrors with Ti nanofilms,

- the optimisation of the guide of the time-of-flight spectrometer IN5,

- a description of the transition guide for the ultra-cold neutron source SuperSUN,

- the evolution of the adaptive focusing technique for extreme conditions developed at PSI,

- and several works towards effective neutron guides simulations.

Eddy Lelièvre-Berna

Editor-in-Chief

\section{References}

[1] B. Jacrot, Des Neutrons Pour la Science, EDP Science, Paris, 2006. ISBN 2-86883-878-2.

[2] T. Springer and A. Heidemann, Maier-Leibnitz and neutron optics, Neutron News 13 (2002), 32-36. doi:10.1080/10448630208222873. 